\title{
Immunolocalisation of Burkholderia cepacia in the lungs of cystic fibrosis patients
}

\author{
UMADEVI SAJJAN, MARY COREY*, ATUL HUMAR†, ELIZABETH TULLIS‡, ERNEST CUTZ\$, \\ CAMERON ACKERLEY§ and JANET FORSTNER
}

Department of Structural Biology and Biochemistry, *Department of Population Health Sciences, Hospital for Sick Children, †Department of Medicine, Toronto General Hospital, †Division of Respiratory Medicine, St Michael's Hospital and §Department of Pediatric Laboratory Medicine and Division of Pathology, Hospital for Sick Children, University of Toronto, Toronto, Ontario, Canada

Infection by Burkholderia cepacia is sometimes fatal in patients with cystic fibrosis (CF), as the organism can cause necrotising pneumonia and septicaemia (the cepacia syndrome), and is resistant to antibiotics. To increase knowledge of the pathogenesis of lung infection, the present study investigated the distribution of $B$. cepacia in lung explants from nine CF recipients of double lung transplants, of which six were colonised with both $B$. cepacia and Pseudomonas aeruginosa and the other three with $P$. aeruginosa only. In one case, explants of the donor lung (allograft) became available after the patient succumbed post-operatively to the cepacia syndrome. Further autopsy sections were examined from two patients who had chronic and then acute infection with $B$. cepacia. A specific antibody reactive with all five genomovars of the $B$. cepacia complex and another antibody specific for the 22-kDa adhesin of cable pili, were used to localise bacteria in situ. In chronic infection, the organisms were diffusely distributed, but most concentrated in hyperplastic bronchiolar epithelium, inflamed peribronchial and perivascular areas, between adjacent airway epithelial cells and in pathologically thickened alveolar septae and luminal macrophages. In acute infections the distribution was more focal, with $B$. cepacia on injured airway surfaces and in sites of pneumonia and abscess formation. In autopsy sections from one of the patients with chronic, then acute infection, $B$. cepacia was also observed in the lumen of blood capillaries. These results suggest that $B$. cepacia has the capacity to be highly invasive, migrating from the airways across the epithelial barrier to invade the lung parenchyma and capillaries, thereby initiating septicaemia.

\section{Introduction}

The aim of the present study was to determine the pattern of distribution of Burkholderia cepacia in the lungs of cystic fibrosis (CF) patients with chronic and acute infections. Valuable insights about the pathogenesis of lung infections due to other organisms have been gained by similarly studying the distribution of bacteria in relation to disease-affected lung structures. For example, it has been demonstrated $[1,2]$ that the location of mucoid Pseudomonas aeruginosa in CF lung is generally endobronchiolar and associated with obliterative changes in small bronchioles. The bacteria were found in regions in which pulmonary damage was

Received 15 Sept. 2000; revised version accepted 23 Nov. 2000.

Corresponding author: Dr J. Forstner (email: jfforst@ sickkids.on.ca). most severe, thus emphasising the association of mucoid $P$. aeruginosa with pulmonary deterioration. $P$. aeruginosa was rarely seen in extraluminal areas, even though inflammatory changes were evident as chronic interstitial infiltration or fibrosis, or both. Staphylococcus aureus, one of the early colonisers in $\mathrm{CF}$ patients, is found mainly in mucus exudates of obstructed airways and not on airway epithelium or in lung parenchyma [3]. In contrast, Haemophilus influenzae is diffusely present in the epithelial and subepithelial layers of bronchi, in large and small bronchioles, in the alveolar epithelium, interstitial tissue, pulmonary macrophages and visceral pleura, but not in the lumen of airways. This widespread distribution is thought to reflect the invasiveness and persistence of $H$. influenzae in CF patients [4]. To date there are no reports documenting the distribution of $B$. cepacia, an opportunist micro-organism that is associated with chronic and acute lung disease in adult and 
adolescent CF patients [5]. At the outset of this study it was not known whether B. cepacia colonises indiscriminately throughout the lung or in focal clusters, in large or small airways, or whether it invades the parenchyma or adjacent extrapulmonary tissues such as pleura or cartilage. All the lung sections for this study came from patients followed in the Toronto CF clinic. In this centre, almost all B. cepacia-infected CF patients harbour one hypertransmissible clonal lineage [6] which belongs to genomovar III of the B. cepacia complex [7]. This strain is known as the ET 12, or RAPD-type 2 strain, and it expresses surface cable pili [8]. Thus, it was anticipated that the distribution patterns of $B$. cepacia in the CF lung would be specific for this strain.

Because B. cepacia is an opportunist pathogen, the development of lung infection in CF patients probably depends on both host and bacterial factors. A study published several years ago described the adherence of cable pili/adhesin-positive genomovar III isolates to mucins [9], implying that hypersecretion of mucus or luminal mucostasis in CF may predispose to chronic colonisation. Prior lung damage due to P. aeruginosa infection may also be a determinant, as it is primarily adolescents and adults with long-standing $P$. aeruginosa lung disease who acquire $B$. cepacia. Various bacterial factors have also been proposed as potential virulence factors, including a potent haemolysin [10], a siderophore [11], surface lipopolysaccharide [12, 13], an extracellular protease [14], lipases [15], a melaninlike pigment [16], as well as the cable pili-associated 22-kDa adhesin which has now been shown to mediate bacterial binding to mucin, cells and lung sections of CF patients [17-20]. The ability of some $B$. cepacia to invade and survive in epithelial cells and macrophages [21-23] and the resistance of this micro-organism to airway epithelial cell antimicrobial activity and to oxidative killing by phagocytes [21,24] may also contribute to virulence.

The present study examined the morphology of lung explants from $\mathrm{CF}$ patients undergoing double lung transplantation (chronic infection), lung allografts from a patient who died due to re-infection with $B$. cepacia shortly after lung transplantation (acute infection) and autopsy specimens from the lungs of patients who were colonised for 5-10 years but were otherwise clinically stable, and then unexpectedly succumbed over a period of 2-3 months to the cepacia syndrome (chronic plus acute infection). B. cepacia was localised in lung sections by immunofluorescent techniques, and its distribution correlated with pathological changes observed in the sections.

\section{Materials and methods}

\section{Patients and tissue samples}

As shown in Table 1, nine CF patients, age-range 1438 years, underwent double lung transplantation at the Toronto Hospital, Ontario between 1989 and 1998. All had been colonised with $P$. aeruginosa at some stage before receiving the transplant and six of them were also colonised with B. cepacia. Of these six, two patients (nos. 5 and 6 ) had had no culturable $P$. aeruginosa in their sputum for 6 and 2 years, respectively, before lung transplantation. Lung explants from patients nos. 1-4 and 7-9 were obtained as paraffin blocks from the Pathology tissue library at the Toronto Hospital. For patients nos. 5 and 6, samples of lung tissue $\left(1 \mathrm{~cm}^{2}\right)$ from both left and right lungs were obtained within $30 \mathrm{~min}$ of explantation and fixed in buffered formalin. Autopsy samples $\left(1 \mathrm{~cm}^{2}\right)$ of lung allografts from patient no. 5 , who died with the cepacia syndrome 11 days after transplantation, were processed in the same fashion. Autopsy lung specimens were obtained from two CF patients (nos. 10 and 11, Table 1) who were colonised with B. cepacia for $>5$ years, then unexpectedly developed the cepacia syndrome and died within a few months. Collection and utilisation of lung samples from patients were approved by the hospital human ethics committee. Paraffin sections ( $5 \mu \mathrm{m}$ thick) were used to determine morphology by haematoxylin and eosin staining.

\section{Bacteria and culture conditions}

All B. cepacia isolates except $\mathrm{BC} 7$ and $\mathrm{BC} 45$ were kindly provided from the $B$. cepacia research panel of

Table 1. Details of patients investigated

\begin{tabular}{|c|c|c|c|c|c|c|c|c|}
\hline \multicolumn{5}{|c|}{ Patient information } & \multirow[b]{2}{*}{ Year transplanted } & \multirow[b]{2}{*}{$\begin{array}{l}\text { First } \mathrm{BC} \text { cultured after } \\
\text { transplantation }\end{array}$} & \multirow[b]{2}{*}{$\begin{array}{l}\text { Survival time } \\
\text { post-transplant }\end{array}$} & \multirow[b]{2}{*}{$\begin{array}{l}\text { Clinical diagnosis } \\
\text { at death }\end{array}$} \\
\hline No. & Age & Sex & $\mathrm{BC}$ & PA & & & & \\
\hline 1 & 21 & M & + & + & 1989 & 5 days & 11 days & Sepsis/pneumonia \\
\hline 2 & 19 & $\mathrm{~F}$ & + & + & 1990 & 2.1 years & 2.3 years & Sepsis/pneumonia \\
\hline 3 & 22 & M & + & + & 1990 & 24 days & 30 days & Sepsis/pneumonia \\
\hline 4 & 35 & M & + & + & 1997 & 5 days & 3 months & Sepsis/pneumonia \\
\hline 5 & 27 & M & + & - & 1998 & 9 days & 11 days & Sepsis/pneumonia \\
\hline 6 & 38 & M & + & - & 1998 & 6 days & Alive & N/A \\
\hline 7 & 23 & M & - & + & 1990 & No BC & Alive & $\mathrm{N} / \mathrm{A}$ \\
\hline 8 & 24 & $\mathrm{~F}$ & - & + & 1996 & No BC & Alive & $\mathrm{N} / \mathrm{A}$ \\
\hline 9 & 14 & $\mathrm{~F}$ & - & + & 1996 & No BC & Lost to follow-up & \\
\hline 10 & 25 & $\mathrm{~F}$ & + & + & \multicolumn{4}{|c|}{ Died with cepacia syndrome 5 years after B. cepacia colonisation (1999) } \\
\hline 11 & 23 & M & + & + & \multicolumn{4}{|c|}{ Died with cepacia syndrome 10 years after $B$. cepacia colonisation (1997) } \\
\hline
\end{tabular}


isolates by $\operatorname{Dr}$ Mahenthiralingam, School of Biosciences, Cardiff University, UK and have been described previously [20]. Isolates BC7 and BC45 were cultured from the sputum of $\mathrm{CF}$ patients at the Hospital for Sick Children and have been characterised previously $[9,17-20]$. Mucoid and non-mucoid $P$. aeruginosa, $H$. influenzae and $S$. aureus were clinical isolates from $\mathrm{CF}$ patients, and were identified and provided by the Department of Clinical Microbiology at the Hospital for Sick Children.

\section{Antibodies}

A rabbit polyclonal antibody (R418) to B. cepacia and a mouse polyclonal antibody (designated anti-adhesin) to the $22-\mathrm{kDa}$ adhesin associated with cable pili of $B$. cepacia have been described previously $[17,20,25]$.

Western blot analyses were done as follows. Bacterial isolates were grown in tryptic soy broth, centrifuged, washed once with phosphate-buffered saline (PBS) and suspended to give $10^{9} \mathrm{cfu} / \mathrm{ml}$. Ten $\mu \mathrm{l}$ from each bacterial suspension were mixed with an equal volume of reducing buffer, boiled for $10 \mathrm{~min}$, centrifuged and the supernate was subjected to SDS-PAGE. Separated proteins were then transferred to a nitrocellulose membrane, blocked with bovine serum albumin (BSA) $3 \%$ in $10 \mathrm{~mm}$ Tris-buffered saline, $\mathrm{pH} 7.6$ (TBS) for $2 \mathrm{~h}$ at room temperature and incubated with the R418 antibody ( 1 in 5000 dilution) overnight at $4^{\circ} \mathrm{C}$. The blot was washed with TBS containing Tween $200.05 \%$ and incubated with a second antibody (antirabbit IgG) conjugated to alkaline phosphatase. For detection, the substrate NBT-BCIP (Boehringer Mannheim, Burlington, Ont., Canada) was used. Specificity of the anti-adhesin antibody was established with crude preparations of outer-membrane proteins from each bacterial species, as described previously [20]. To establish further the specificity of R418 and the antiadhesin antibody, and to ensure that both antibodies would detect bacterial cells in lung sections, bacterial smears prepared on glass slides were incubated with 1 in 1000 diluted R418 antibody or 1 in 100 diluted antiadhesin antibody for $1 \mathrm{~h}$ at $37^{\circ} \mathrm{C}$. Slides were washed three times with TBS and incubated with appropriate anti-rabbit or anti-mouse $\operatorname{IgG}$ conjugated to $\mathrm{CY} 3$ fluorophore ( 1 in 250 dilution). Bacteria were observed by light and fluorescence microscopy (Leica Dialux 22, Willowdale, Ont., Canada). Bacterial smears and lung sections treated with non-immune serum or immune serum pre-absorbed with cable pili/adhesin-positive $B$. cepacia served as controls.

\section{Immunolocalisation of B. cepacia in lung sections}

Paraffin sections of lung tissue were mounted on silanated slides, deparaffinised by xylene treatment, rehydrated in graded alcohols, washed twice with PBS and treated with Triton X-100 $0.1 \%$ in PBS for $10 \mathrm{~min}$ at room temperature. Some sections required proces- sing by microwave heating (DAKO Diagnostics Cana$\mathrm{da}$, Mississauga, Ont., Canada) for $1.5 \mathrm{~min}$ in $10 \mathrm{~mm}$ citrate buffer, $\mathrm{pH} 6.0$ [26] for detection of B. cepacia in the airway lumen. Sections were rinsed with water, equilibrated with TBS, blocked with goat serum $5 \%$ in TBS for $1 \mathrm{~h}$ at room temperature and then incubated for $1 \mathrm{~h}$ with the R418 (1 in 1000 dilution) or antiadhesin ( 1 in 100 dilution) antibody. Unbound antibody was removed by washing with TBS three times (10 min each) and sections were then incubated with anti-rabbit or anti-mouse IgG conjugated to CY3 fluorophore as appropriate. Slides were washed three times with TBS as before, counter-stained with Mayer's haematoxylin (Sigma) and examined with a fluorescence microscope or by confocal microscopy (Leica TCS 4D equipped with Argon-Krypton laser scanner; Heidelburg, Germany). Optical sectioning (c. $1 \mu \mathrm{m}$ thickness) of lung sections was performed in the $\mathrm{X}-\mathrm{Y}$ plane. The bacterial load of B. cepacia-colonised lung was semiquantified by observing 15 random fields in each section and grading each section 1-4 depending on the number of fields that were positive for $B$. cepacia. The presence of $B$. cepacia in 11-15 fields was given a value of 4 ; in $6-10$ fields a value of 3 ; in $3-5$ fields a value of 2; and in 1-2 fields a value of 1 . Scores from all sections for each patient were averaged to arrive at a final score. Control samples were treated with nonimmune rabbit or mouse serum, or primary antibody absorbed with B. cepacia isolate BC7.

\section{Immuno-electron microscopy}

Lung allografts fixed in formalin $10 \%$ were washed with PBS, fixed in paraformaldehyde $4 \%$ and glutaraldehyde $0.1 \%$ in $0.1 \mathrm{M}$ phosphate buffer and embedded in Lowicryl (K4M). Thin sections were taken and mounted on formvar-coated nickel grids, blocked with PBS containing glycerol $0.15 \%$ and BSA $2 \%$ and then incubated with 1 in 1000 diluted R418 or 1 in 100 diluted anti-adhesin antibody for $1 \mathrm{~h}$ at room temperature. Sections were rinsed with PBS containing BSA to remove unbound antibody and then incubated with anti-rabbit or anti-mouse immunoglobulin conjugated with $10 \mathrm{~nm}$ colloidal gold ( 1 in 20 dilution) for $1 \mathrm{~h}$ at room temperature. Sections were rinsed with distilled water, counter-stained with uranyl acetate and lead acetate, and observed with a JEOL 1200 II transmission electron microscope (JEOL USA, Peabody, MA, USA) fitted with a lanthanum hexaboride cathode and operated at $60 \mathrm{LV}$ accelerating voltage.

\section{Results}

\section{Specificity of $R 418$ and 22-kDa adhesin antibodies}

Western blot analyses were performed on extracts (lysates) and crude outer-membrane preparations of $B$. cepacia isolates belonging to all five genomovars, and clinical isolates of other common CF pathogens (Fig. 
1). The R418 antibody reacted strongly with numerous bands in all B. cepacia isolates tested (Fig. 1a) but not with $P$. aeruginosa, $H$. influenzae or $S$. aureus. The anti-adhesin antibody reacted only with genomovar III isolates BC7 and C5424 (Fig. 1b). The other three genomovar III isolates lack the adhesin $[17,20]$ and did not react with the antibody.

The reactivity of both antibodies with intact bacteria was also tested by immunofluorescent assays of bacterial smears (data not shown). The R418 antibody reacted strongly with all $B$. cepacia isolates but not with other micro-organisms, indicating that the R418 antibody would be appropriate for detection of $B$. cepacia in sections of lung tissue. The anti-adhesin antibody reacted strongly with the two cable pili/ adhesin-positive B. cepacia isolates BC7 and C5424, very faintly with all other $B$. cepacia isolates and not at all with other micro-organisms. These results predicted that the antibody would detect cable pili-positive isolates expressing the $22-\mathrm{kDa}$ adhesin in lung tissue sections.

\section{Lung explants of CF patients (chronic infection with B. cepacia)}

At least one section from each lung transplant patient except patient no. 3 (Table 1) was taken from the proximal part of the lung, which showed second to fifth generation bronchi and large bronchioles. Other sections (four-to-eight per patient) were representative of peripheral lungs, showing numerous small, terminal and respiratory bronchioles and adjacent parenchyma. Some sections also contained pleural membrane. All

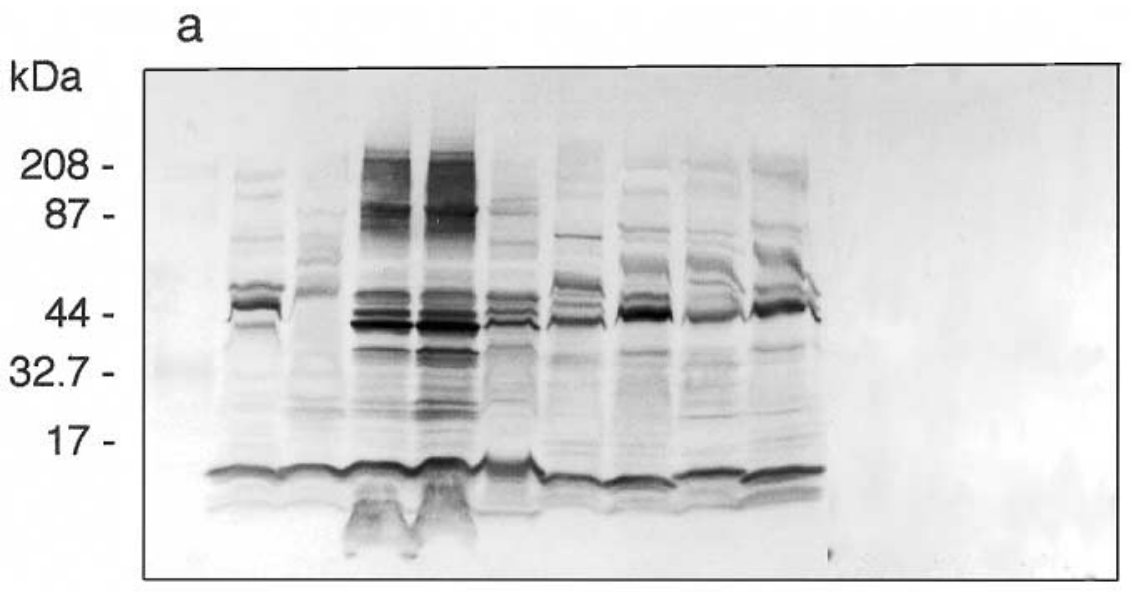

$\begin{array}{llllllllllllll}1 & 2 & 3 & 4 & 5 & 6 & 7 & 8 & 9 & 10 & 11 & 12 & 13 & 14\end{array}$

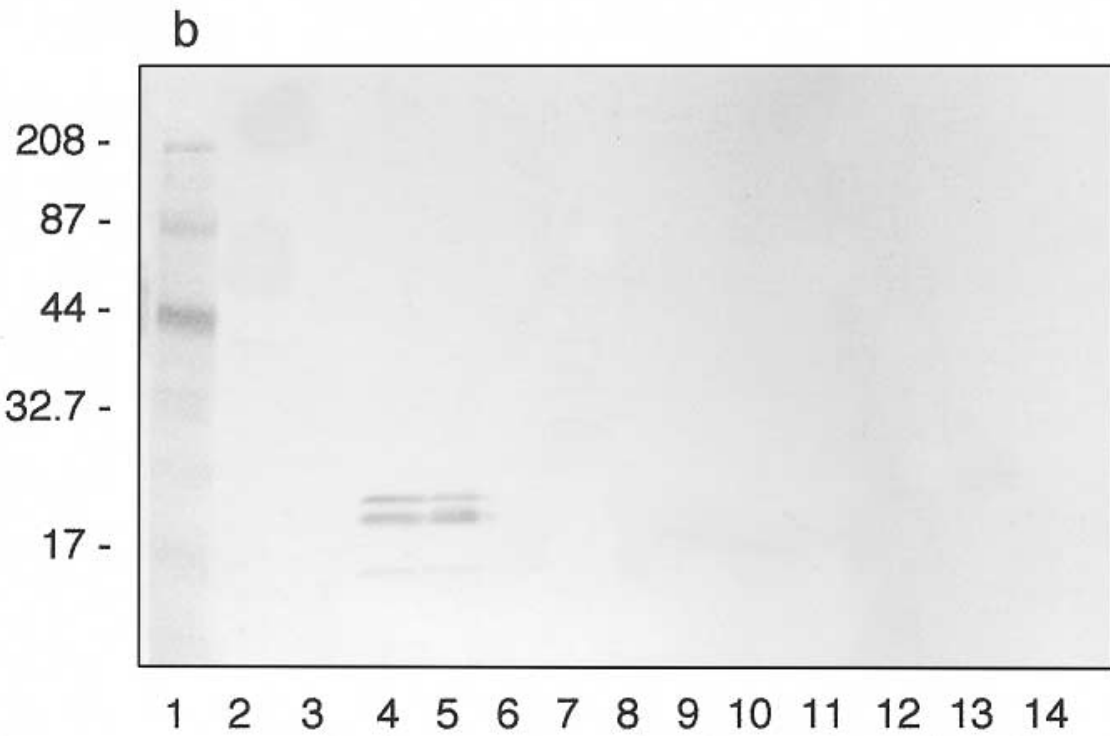

Fig. 1. Immunoreactivity of R418 and anti-adhesin $(22 \mathrm{kDa})$ antibody with B. cepacia and other CF lung pathogens. Bacterial extracts (a) or crude outer-membrane preparations (b) from B. cepacia (genomovars I, II, III, IV and V) and other organisms $\left(10^{7} \mathrm{cfu}\right)$ were subjected to SDS-PAGE and proteins were transferred to a nitrocellulose membrane. The membrane was incubated with antibody R418 (a) or the anti-adhesin antibody (b). Lane 1, molecular mass standards; 2, B. cepacia ATCC 25416 (genomovar I); 3, C5393 (genomovar II); 4, BC7; 5, C5424; 6, BC45; 7, J2315; 8, CEP138 (genomovar III); 9, FC473 (genomovar IV); 10, CEP040 (genomovar V); 11-14, clinical isolates of nonmucoid $P$. aeruginosa, mucoid $P$. aeruginosa, $S$. aureus and $H$. influenzae, respectively. 
lung explants showed evidence of end-stage lung disease, including marked bronchiectasis and extensive mucus plugging (Fig. 2a). Within the mucus plugs, a dense infiltrate of acute and chronic inflammatory cells, plasma cells and exfoliated epithelial cells was noted. Often neutrophils were also seen within the bronchial/bronchiolar epithelial layer. Hyperplasia, squamous metaplasia, partial loss of cilia or exfoliation of epithelia, or both, were observed in many small bronchioles. Peribronchiolar and perivascular fibrosis with focal interstitial extension was seen in many sections. Many alveolar septae were abnormally thickened (Fig. 2b) with loose fibrous connective tissue and infiltrating macrophages. In some sections interlobular septae had dilated lymphovascular spaces. Many of the air spaces contained scattered macrophages. Samples from all the patients showed many regions of pneumonic consolidation with heavy infiltration by neutrophils. By visual inspection no difference was observed in morphology between patients colonised with $P$. aeruginosa alone (patients nos. 7, 8 and 9, Table 1) and those colonised with both $P$. aeruginosa (either in the past or at the time of transplant) and B. cepacia (patients nos. 1-6, Table 1).
Immunofluorescence of $B$. cepacia in lung explants was performed with antibody R418 and the antiadhesin antibody. Two different methods were used to detect the colonised organisms in paraffin-embedded tissue sections. In the first method, sections were treated with Triton X-100 $0.1 \%$ which permeabilises tissue and exposes antigenic sites. In the second method, sections were heated in a microwave oven to 'retrieve' antigens [26]. Both methods gave comparable results in all patients except where indicated below. Both antibodies gave positive signals only in explants from B. cepacia-infected patients (nos. 1-6) and not in explants from patients who were colonised with only $P$. aeruginosa (nos. 7-9). The load of colonised $B$. cepacia differed between patients (Table 2), but no correlations could be discerned between the apparent load, the degree of pathology or the subsequent outcome of double lung transplantation in these patients. Lung explant sections treated with nonimmune serum, or R418 antibody pre-incubated with B. cepacia (controls), were negative.

B. cepacia was found in both the airways and the lung parenchyma of patients nos. 1-6, principally in
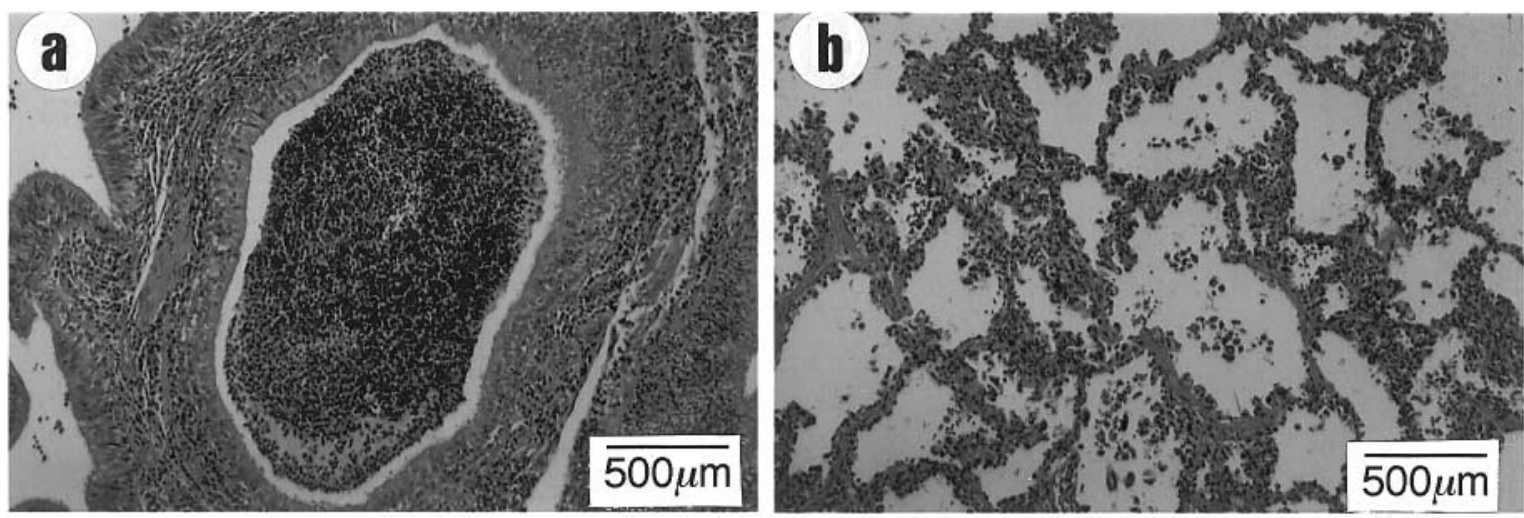

Fig. 2. Haematoxylin and eosin-stained sections of a CF lung explant from a B. cepacia colonised patient. (a) Mucus exudate plugging a small bronchiole; (b) thickened alveolar septa. Both sections show extensive inflammatory cell infiltration.

Table 2. Distribution of $B$. cepacia in CF explant and autopsy lungs

\begin{tabular}{|c|c|c|c|c|c|c|c|c|c|c|}
\hline \multirow[b]{3}{*}{ Patient no. } & \multicolumn{10}{|c|}{ Grade of $B$. cepacia colonisation detected by immunofluorescence in } \\
\hline & \multicolumn{2}{|c|}{$\begin{array}{c}\text { Bronchiolar (small) } \\
\text { epithelium }\end{array}$} & \multicolumn{2}{|c|}{ Alveolar septum } & \multicolumn{2}{|c|}{ Bronchial epithelium } & \multicolumn{2}{|c|}{ Airway (bronchi) lumen } & \multicolumn{2}{|c|}{ Cartilage } \\
\hline & $\mathrm{R} 418$ & $22 \mathrm{kDa}$ & R418 & $22 \mathrm{kDa}$ & R418 & $22 \mathrm{kDa}$ & R418 & $22 \mathrm{kDa}$ & R418 & $22 \mathrm{kDa}$ \\
\hline 1 & 4 & 4 & 4 & 4 & 1 & 1 & 2 & 2 & 2 & 0 \\
\hline 2 & 4 & 1 & 4 & 1 & 4 & 1 & 4 & 1 & 4 & 0 \\
\hline 3 & 3 & 3 & 4 & 4 & N/A & N/A & N/A & N/A & N/A & N/A \\
\hline 4 & 4 & 4 & 4 & 4 & 1 & 1 & 1 & 1 & 0 & 0 \\
\hline 5 & 2 & 2 & 3 & 3 & 1 & 1 & 1 & 1 & 0 & 0 \\
\hline 6 & 2 & 2 & 2 & 2 & 1 & 1 & 1 & 1 & 0 & 0 \\
\hline 10 & 4 & 4 & 4 & 4 & N/A & N/A & N/A & N/A & N/A & N/A \\
\hline 11 & 4 & 4 & 4 & 4 & 4 & 4 & 4 & 4 & 4 & 4 \\
\hline
\end{tabular}

Bacteria detected by immunofluorescence with $\mathrm{R} 418$ or anti-adhesin $(22 \mathrm{kDa})$ antibody. Localisation of $B$. cepacia was scored over 15 random high-power fields. Scoring: presence of $B$. cepacia in $11-15$ fields $=4 ; 6-10$ fields $=3 ; 3-5$ fields $=2$ and $1-2$ fields $=1$. Patients nos. $7-9$ are not presented as they were colonised only by $P$. aeruginosa and gave no reactivity in any location with anti-B. cepacia antibodies. N/A, not available. 
peripheral rather than proximal airways. In the larger bronchi (second to fifth generation), B. cepacia clusters were occasionally observed associated with epithelium and luminal exudates, as judged by the tissue permeabilisation method. In small bronchi (Fig. 3a and b), B. cepacia was found in surface epithelial cells, but even more prominently in the deeper layers of hyperplastic thickened respiratory epithelium. This preferential deeper distribution has not been described for other CF pathogens. Patient no. 2 showed abundant immunoreactive B. cepacia in the epithelium of small bronchi, but very few bacteria in the lumen, even though numerous gram-negative rods were identified by Gram's staining. This prompted the use of the antigen retrieval technique instead of Triton X-100 permeabilisation of tissue sections, before incubating with the R418 antibody. With antigen retrieval, numerous $B$. cepacia were detected in the exudate of the bronchial lumen (Fig. 3c and d), but bacteria in the epithelium were less well detected by this technique. It was concluded that permeabilisation of the tissue is necessary to visualise bacteria associated with the epithelium, whereas the antigen retrieval technique optimises visualisation of $B$. cepacia in the lumen. Proximal lung sections from several patients were then re-tested by the latter technique and, in most sections, very few B. cepacia were found in the lumen of large or small bronchi. However, positive signals were observed in the lumen of bronchioles that also contained mucopurulent exudates.

Colonisation was not confined to grossly inflamed bronchioles, which is the situation reported for $P$. aeruginosa $[1,2]$. Frequently, B. cepacia (or, possibly,
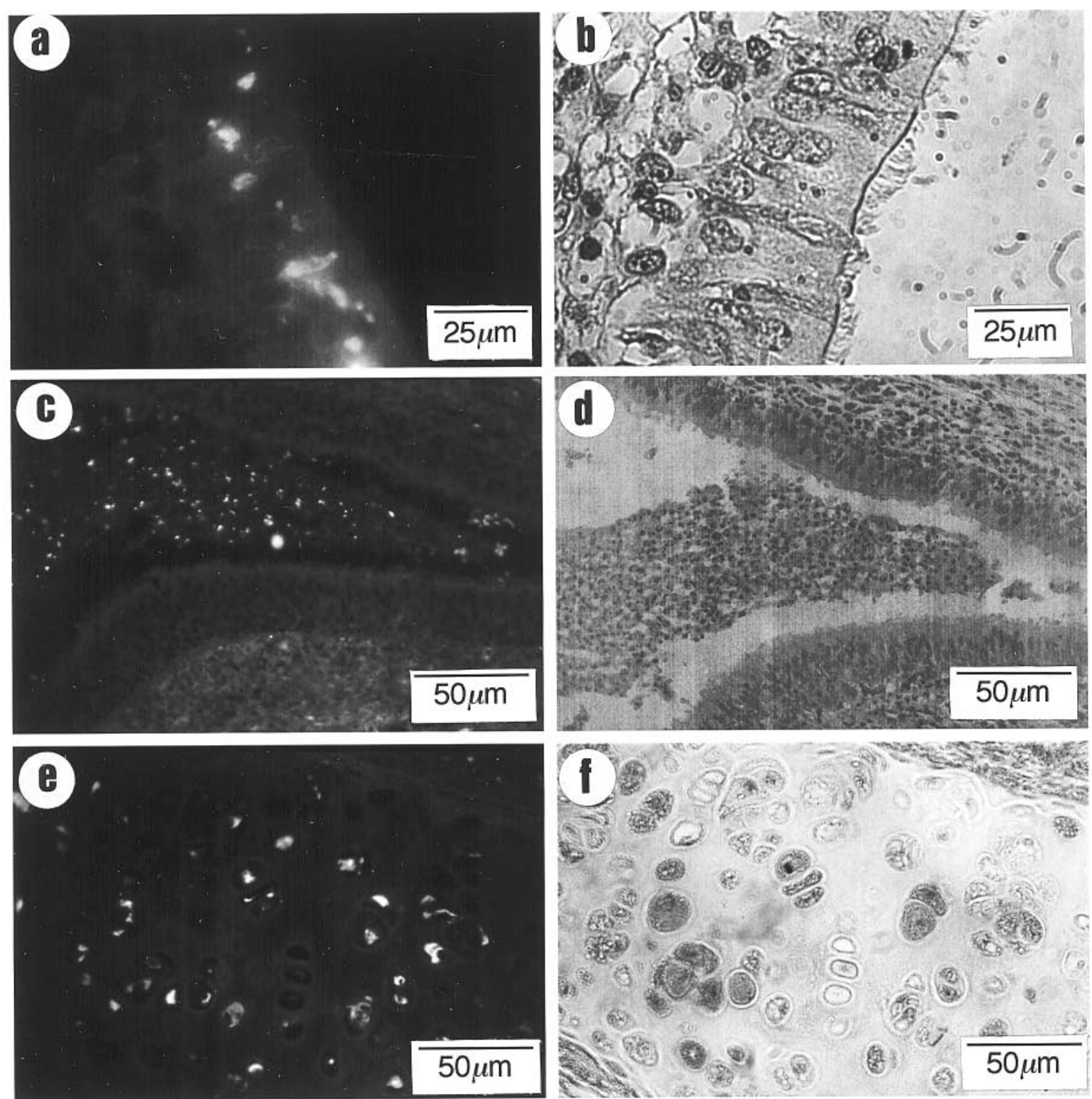

Fig. 3. Distribution of B. cepacia in small bronchi of CF lung explants. Treated paraffin sections were incubated with R418 antibody followed by anti-rabbit IgG conjugated with fluorophore. (a), (c) and (e) show immunoreactive $B$. cepacia in the bronchial epithelium, lumen and cartilage, respectively; (b), (d) and (f) show haematoxylin-stained sections corresponding to (a), (c) and (e) respectively. 
immunoreactive degradation products of the organism) were observed in the intercellular junctions of epithelial cells of relatively normal-looking bronchioles (Fig. $4 \mathrm{a}$ and $\mathrm{b}$ ). This was best revealed by high resolution confocal microscopy with optical sections taken every micrometer, and reconstruction of the image (Fig. 4c). The bacteria were detected by both antibodies, indicating that they belonged to the cable/adhesin-positive ET 12 strain. Intercellular distribution has not been described for other CF pathogens and suggests an invasive phenotype. B. cepacia was also detected in peribronchiolar regions containing inflammatory cell infiltrates (Fig. 4d and e), suggesting that the bacteria may be able to migrate from the lumen via intercellular pathways to the deeper parenchyma.
Unlike the reported distribution of $P$. aeruginosa in $\mathrm{CF}$ lungs, B. cepacia was prominent in abnormally thickened alveolar septae in explants from all six patients (Fig. 5a and b) and was associated with alveolar epithelial cells, infiltrating and luminal macrophages (Fig. 5c and d). Pulmonary tissue that had been replaced by consolidated inflammatory cells frequently showed abundant immunoreactive B. cepacia (Fig. 5e and f), although fibrotic tissue was spared.

Surprisingly, explants from patients nos. 1 and 2 also showed B. cepacia in the cartilage adjacent to small bronchi. The micro-organisms were associated with calcified chondrocytes (Fig. 3e and $\mathrm{f}$ ) and, in patient no. 1, B. cepacia was also noted in the submucosal
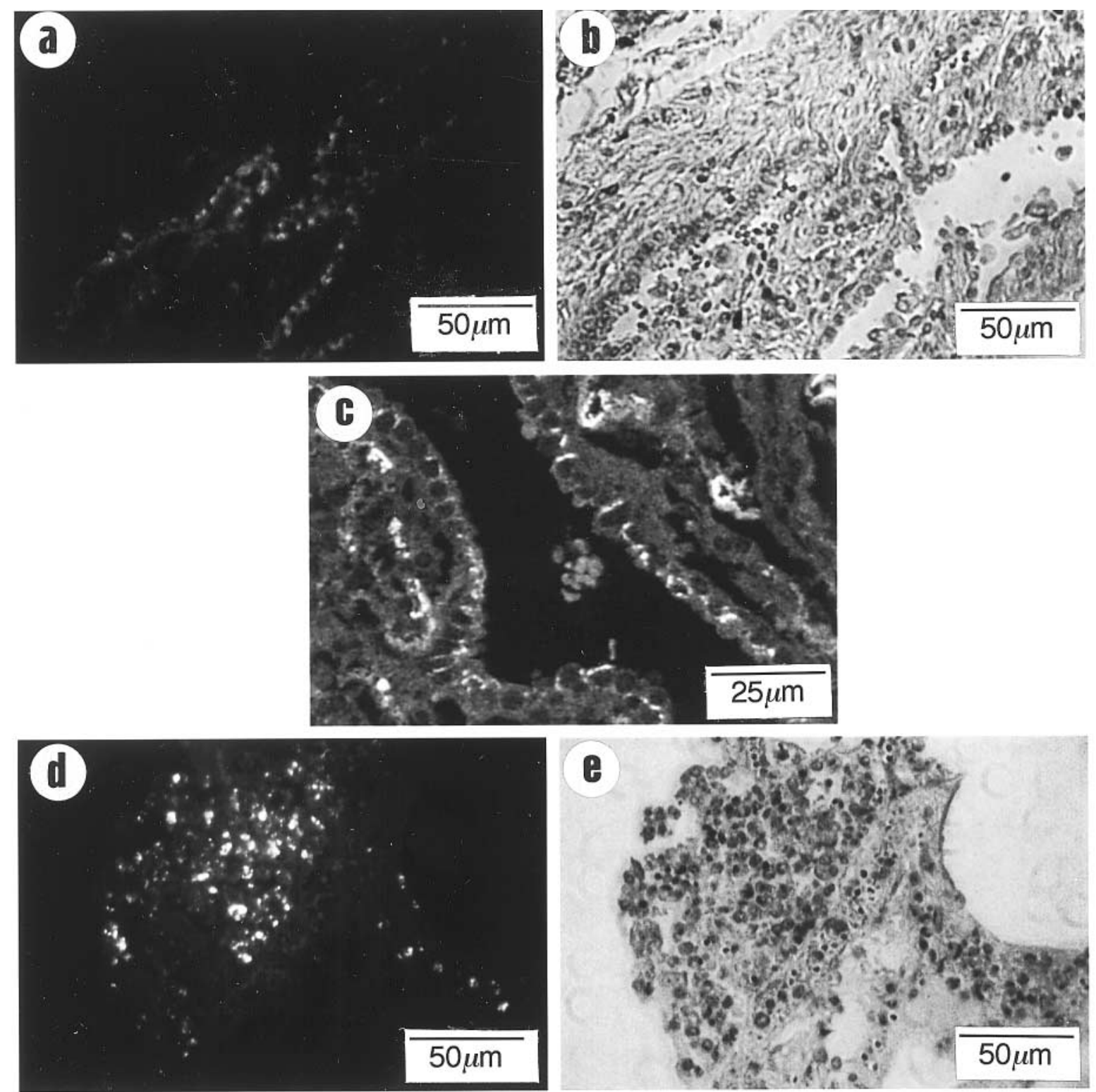

Fig. 4. Distribution of B. cepacia in terminal bronchioles and peribronchiolar areas of CF lung explants. Deparaffinised and rehydrated sections incubated with antibody R418 (a) and (d) or anti-adhesin antibody (c). (a) and (d) B. cepacia in a terminal bronchiole and a peribronchiolar area; (b) and (e) haematoxylin-stained sections corresponding to (a) and (d) respectively; (c) high resolution confocal image of a terminal bronchiole showing B. cepacia distributed in the junctions between adjacent epithelial cells and in the parenchyma. 

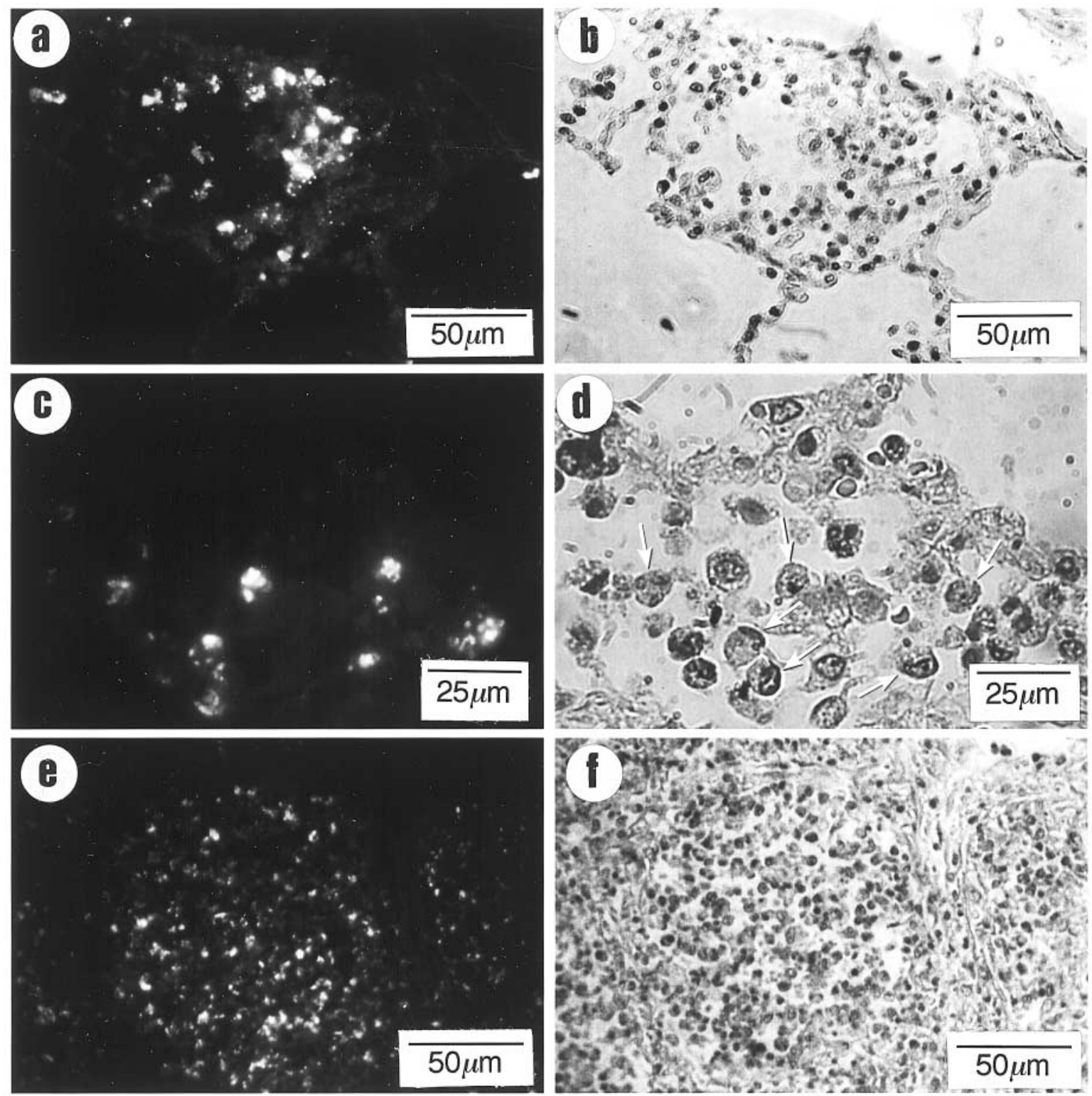

Fig. 5. Distribution of $B$. cepacia in the parenchyma of CF lung explants reactive with anti-adhesin antibody. (a) $B$. cepacia in alveolar septa, (c) association of B. cepacia with alveolar macrophage, (e) B. cepacia distribution in consolidated area; (b) (d) and (f) are haematoxylin-stained sections corresponding to (a), (c) and (e), respectively; (d) $B$. cepacia associated with alveolar macrophages are shown by arrows.

glands. These are unusual locations for a pulmonary pathogen, but recently $H$. influenzae has also been detected in cartilage cells and submucosal glands of $\mathrm{CF}$ patients [4].

By visual inspection, the distribution and load of $B$. cepacia revealed by the anti-adhesin antibody were the same as those revealed by the R418 antibody in the lung explants of all but two B. cepacia-colonised patients. The anti-adhesin antibody failed to detect $B$. cepacia in chondrocytes (patients nos. 1 and 2) and the anti-adhesin signal was much lower than the R418 antibody signal in all tissues of patient no. 2. This suggests a loss or masking of the adhesin of B. cepacia once the bacteria migrate to cartilage cells and a similar loss of adhesin in the B. cepacia of patient no.
2. Alternatively there may have been colonisation of the lungs of patient no. 2 by more than one strain (or genomovar), with the dominant strain lacking cable pili. Unfortunately, sputum cultures were not available to distinguish between these alternatives.

Taken together, these results suggest that the majority of $B$. cepacia in the lungs of chronically infected Toronto patients (nos. 1-6) belonged to genomovar III, and were the ET 12 or cable pili/adhesin-positive strain. The bacteria were mainly distributed distally, on the surface and between epithelial cells of small bronchioles, in the deeper layers of hyperplastic epithelia, and within the chronically inflamed alveolar septae. This distribution is clearly consistent with an invasive profile. 


\section{Lung allografts of a CF patient (acute infection of the donor lung)}

Allograft tissue was available from only one patient (no. 5). The broncho-alveolar lavage fluid was $B$. cepacia culture-positive 2 days after double lung transplantation. Subsequently, the patient developed bronchopneumonia and sepsis, and died 9 days later. Gross pathological observation of the lungs indicated focal areas of necrotising bronchopneumonia and pleural effusion, but many regions of both lungs appeared normal.

Sections from infected regions showed necrotising pneumonia, consolidation, micro-abscesses and no normal airway or parenchymal structures. A bronchial section close to the site of surgical anastomosis showed partly exfoliated epithelium with some inflammatory cells in the lumen. Sections obtained from minimally inflamed areas also showed partially exfoliated epithelium in bronchioles and cellular material in the lumen. Although alveolar septae appeared normal, the alveolar air space was filled with inflammatory cells, mainly foamy macrophages. Non-inflamed, normal-appearing areas showed normal lung histology. The alveolar septae were also normal, but the alveolar airspaces frequently showed foamy alveolar macrophages, and occasionally neutrophils, suggestive of a low grade inflammatory reaction.

Immunofluorescent localisation of B. cepacia produced strong signals from both antibodies in regions of pneumonia, exfoliating epithelium close to the anastomosis site (Fig. 6a and b) and in perivascular areas containing inflammatory infiltrates (Fig. 6c and d). Signals were not seen in the lumen, on the surface of bronchiolar epithelia, or in alveolar septae in normal areas of the lung. However, micro-organisms reactive with both antibodies were found associated with foamy macrophages in otherwise normal-appearing alveolar airspaces (Fig. 6e). Electron microscopy confirmed that B. cepacia cells were inside macrophages (Fig. 6f, shown by arrows), and had retained the immunoreactive adhesin (Fig. 6 inset, gold labelling shown by arrowheads).

\section{Lungs from CF patients who died with a 'delayed' cepacia syndrome (chronic plus acute infection with B. cepacia)}

In this group there were two patients (nos. 10 and 11) who were infected chronically with B. cepacia for $>5$ years, then developed the cepacia syndrome and died within 3 months. Their lungs showed extensive necrotising pneumonia and abscess formation, diffuse alveolar damage and interstitial pneumonia. In most sections the airways and parenchyma were not easily recognised because of massive tissue necrosis. Some sections showed recognisable airways, but they were denuded of epithelium and the lumen was filled with exudate and inflammatory cells. Alveolar septae were thickened and infiltrated with foamy macrophages. The air spaces also often contained macrophages. Tomashefski et al. [27] reported similar histopathological changes in the lungs of CF patients who had a rapid decline with fatal necrotising pneumonia and septicaemia shortly after B. cepacia colonisation.

Both antibodies gave an identical pattern of distribution. The bacterial load was scored as 4 in the same scoring system as that used for explants (Table 2). Numerous B. cepacia cells were detected in regions of interstitial pneumonia and abscesses, in damaged epithelial cell layers of bronchi and in alveolar macrophages in air spaces (not shown). B. cepacia was also found in small, terminal and respiratory bronchioles and in alveolar epithelia. One of the patients (no. 10) also had B. cepacia in capillaries adjacent to alveoli (Fig. 7), a location not seen either in lung explants or allograft sections. Thus the autopsy sections exhibited B. cepacia distribution characteristics that were a combination of acute and chronically infected lungs, but in addition, there was evidence of intravascular invasion.

\section{Discussion}

Although the prevalence of $B$. cepacia lung infection in $\mathrm{CF}$ patients is estimated to be only about $3 \%$ on a world-wide scale [5], it is much higher in selected geographic regions, including Toronto, Canada, where it has been found in sputum cultures of $>40 \%$ of the adult CF population [28]. The clinical outcome after acquisition is variable and unpredictable, ranging from no apparent change to a rapidly fatal necrotising pneumonia and septicaemia (the cepacia syndrome) [29-31]. Infected patients who undergo double lung transplantation have a particularly high risk of infection in the donor lung and death due to the cepacia syndrome during the early post-operative period, when they are maintained on heavy doses of immunosuppressive drugs. The result is a much higher mortality rate in the first 3 months post-operatively (64\%) than for transplant patients previously colonised by $P$. aeruginosa alone $(19 \%)$ [32,33]. Results from the present study indicate that cable pili/adhesin-positive $B$. cepacia can be highly invasive, migrating from the airways across the epithelial barrier, to invade the lung parenchyma and blood vessels. This may help to explain why $\mathrm{CF}$ patients colonised with $B$. cepacia before undergoing double lung transplantation are at high risk for post-operative mortality.

The distribution of B. cepacia was determined in explants from patients with chronic and acute infections, with and without concomitant $P$. aeruginosa infections. It was considered important to use antisera with multiple recognition epitopes to prevent misidentification of B. cepacia, or lack of recognition of $B$. 

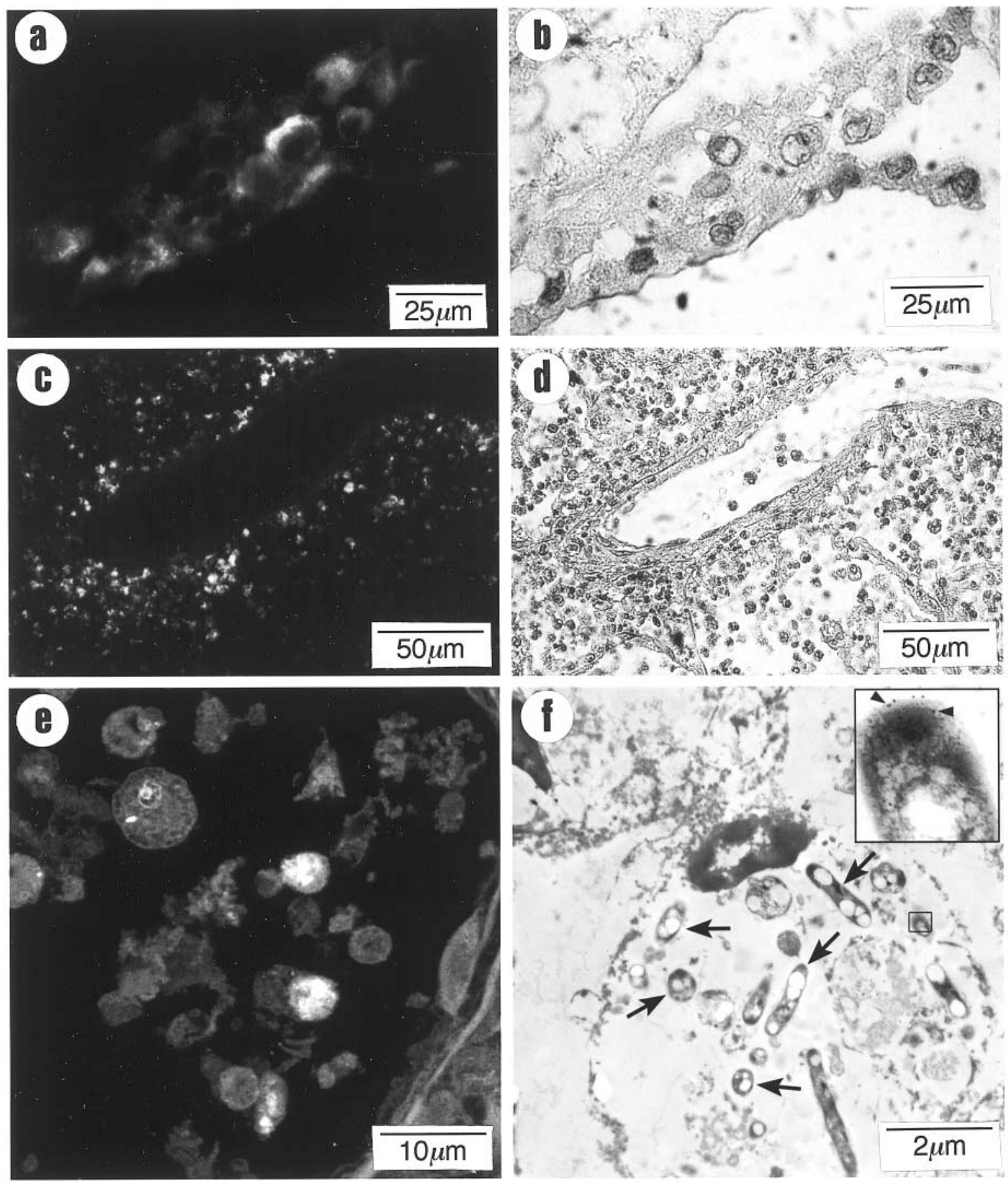

Fig. 6. B. cepacia distribution in lung allografts detected with anti-adhesin antibody. (a) B. cepacia in a small bronchus with exfoliating epithelia; (c) B. cepacia in an inflamed perivascular area; (b) and (d) haematoxylin-stained sections corresponding to (a) and (c), respectively; (e) B. cepacia in alveolar macrophages (confocal microscopy); (f) transmission electron microscopy of a lung allograft section with bacteria (arrows) in a macrophage. The inset is a portion of one bacterium (magnification $14 \times$ ) showing colloidal gold immunoreactivity with the anti-adhesin antibody (arrowheads).

cepacia that might have lost antigenic epitopes during in-vivo colonisation. The R418 antibody recognised multiple protein bands in all five genomovars tested, whereas the anti-adhesin antibody was highly specific for the 22-kDa doublet previously identified in cable pili-positive genomovar III organisms [20]. In all but one patient (no. 2), the immunoreactivities of $B$. cepacia detected in lung tissue by both antisera were identical, suggesting that the strain responsible for infection was the cable pili/adhesin-positive genomovar
III strain. Further specificity was confirmed by demonstrating that other common respiratory pathogens did not react with the antibodies, that the antibodies did not react with lung explants taken from patients colonised by $P$. aeruginosa alone, and that preadsorption of R418 antibody with isolate BC7 (cable/ adhesin-positive $B$. cepacia) abrogated reactivity. In the case of patient no. 2, B. cepacia cells reactive with antibody R418 were more numerous than those reactive with the anti-adhesin antibody. It is likely that this 


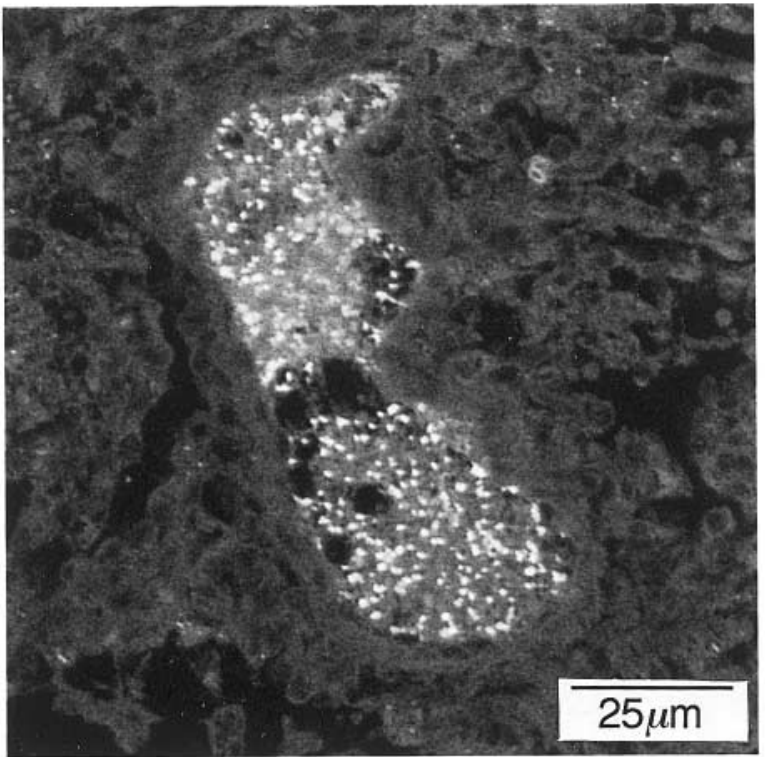

Fig. 7. B. cepacia in an alveolar capillary of a CF patient who died with the delayed cepacia syndrome. Bacteria were detected with the anti-adhesin antibody.

reflects colonisation by more than one strain of $B$. cepacia, an unusual but previously recognised occurrence in CF [5,34]. Alternatively, some members of the cable pili-positive strain may have undergone phase variation with a loss of the pili and associated adhesin. Loss of pili is a frequent occurrence in selected strains of Escherichia coli and H. influenzae once they have colonised host tissues $[35,36]$. As five of the six $B$. cepacia-infected patients did not appear to lose adhesin reactivity, phase variation seems an unlikely explanation. One possible exception might be in the case of the B. cepacia noted in calcified chondrocytes of both patients nos. 1 and 2, as neither reacted with the antiadhesin antibody in that location.

The morphology of CF lung tissues was similar in all nine explants studied, with no distinctive features peculiar to B. cepacia-infected patients. This is not surprising considering that the patients all had endstage lung disease and widespread destruction of normal lung structure at the time the explants were obtained. However, the distribution of B. cepacia was much more widespread and invasive in all cases than that reported for $P$. aeruginosa $[1,2]$. B. cepacia was found mainly in epithelia of the terminal and respiratory bronchioles, and exhibited intercellular migration, presumably allowing it to penetrate the respiratory epithelium. The mechanism permitting penetration was not explored, but it might be speculated that bacterial exoproducts (proteolytic enzymes) could be involved. Organisms were also numerous in peribronchiolar and perivascular regions, in inflammatory cell infiltrates, in thickened alveolar septae and in luminal and parenchymal macrophages. These are not typical locations for $P$. aeruginosa, which tend to localise to luminal positions but do not persist in phagocytes. Overall, the B. cepacia distribution was closer to that described by others for $H$. influenzae [4], although the intercellular location of $B$. cepacia appears to be unique.

The allograft of patient no. 5 who died 11 days after transplantation, and the autopsy sections of patients nos. 10 and 11, who exhibited a 'delayed' cepacia syndrome, showed areas of necrotising pneumonia, unlike the explant tissues from chronically infected patients. This is consistent with previous descriptions of lung tissue from patients who succumbed to the cepacia syndrome shortly after colonisation [27]. $B$. cepacia was prominent in areas of necrotising pneumonia, and a special feature of acute infections was the high number of 'foamy' macrophages in alveolar spaces, many of which contained cable pili/adhesinpositive organisms. B. cepacia is known to be able to persist in $\mathrm{CF}$ macrophages, at least under in-vitro conditions [21,22], and to replicate and resist killing by an oxidative burst. This may be an important mechanism in vivo to allow B. cepacia to escape the normal immune clearance process.

The septicaemia characteristic of the cepacia syndrome was dramatically revealed by autopsy sections from patient no. 10 in which immunoreactive organisms were seen to fill the capillary lumen, and by the allograft sections from patient no. 5 in which perivascular areas were particularly prominent sites of bacterial clusters. Thus, it is speculated that cable pili/ adhesin-positive $B$. cepacia can be highly invasive, especially in immunocompromised patients, moving from the airways through the epithelial barrier to parenchyma and into blood capillaries of the donor lung within a few days post-operatively. Other CF lung pathogens rarely, if ever, exhibit such rapidly invasive behaviour, or lead to such devastating results as those seen in the cepacia syndrome.

We are indebted to the following for assistance in providing clinical samples and information: Dr D. Chamberlain, Division of Pathology, Dr S. Keshavjee, Division of Thoracic Surgery, Toronto General Hospital; Dr D. Battaglia, Division of Pathology, St Michael's Hospital, Toronto and Dr J. Yankaskas, Division of Pulmonary and Critical Care Medicine, Department of Medicine, University of North Carolina at Chapel Hill, Chapel Hill, NC, USA. Financial support was received from the Canadian Cystic Fibrosis Foundation.

\section{References}

1. Baltimore RS, Christie CDC, Smith GJW. Immunohistopathologic localization of Pseudomonas aeruginosa in lungs from patients with cystic fibrosis. Implications for the pathogenesis of progressive lung deterioration. Am Rev Respir Dis 1989; 140: $1650-1661$.

2. Potts SB, Roggli VL, Spock A. Immunohistologic quantification of Pseudomonas aeruginosa in the tracheobronchial tree from patients with cystic fibrosis. Pediatr Pathol Lab Med 1995; 15: 707-721.

3. Ulrich $\mathrm{M}$, Herbert $\mathrm{S}$, Berger $\mathrm{J}$ et al. Localization of Staphylococcus aureus in infected airways of patients with cystic fibrosis and in a cell culture model of $S$. aureus 
adherence. Am J Respir Cell Mol Biol 1998; 19: 83-91.

4. Möller LVM, Timens W, van der Bij W et al. Haemophilus influenzae in lung explants of patients with end-stage pulmonary disease. Am J Respir Crit Care Med 1998; 157: 950-956.

5. LiPuma JJ. Burkholderia cepacia. Management issues and new insights. Clin Chest Med 1998; 19: 473-486.

6. Sun L, Jiang R-Z, Steinbach S et al. The emergence of a highly transmissible lineage of cbl+ Pseudomonas (Burkholderia) cepacia causing CF centre epidemics in North America and Britain. Nature Med 1995; 1: 661-666.

7. Vandamme P, Holmes B, Vancanneyt M et al. Occurrence of multiple genomovars of Burkholderia cepacia in cystic fibrosis patients and proposal of Burkholderia multivorans sp. nov. Int J Syst Bacteriol 1997; 47: 1188-1200.

8. Sajjan US, Sun L, Goldstein R, Forstner JF. Cable (Cbl) type II pili of cystic fibrosis-associated Burkholderia (Pseudomonas) cepacia: nucleotide sequence of the $c b l \mathrm{~A}$ major subunit pilin gene and novel morphology of the assembled appendage fibers. $J$ Bacteriol 1995; 177: 1030-1038.

9. Sajjan US, Corey M, Karmali MA, Forstner JF. Binding of Pseudomonas cepacia to normal human intestinal mucin and respiratory mucin from patients with cystic fibrosis. $J$ Clin Invest 1992; 89: 648-656.

10. Hutchison ML, Poxton IR, Govan JR. Burkholderia cepacia produces a hemolysin that is capable of inducing apoptosis and degranulation by mammalian phagocytes. Infect Immun 1998; 66: $2033-2039$

11. Darling P, Chan M, Cox AD, Sokol PA. Siderophore production by cystic fibrosis isolates of Burkholderia cepacia. Infect Immun 1998; 66: 874-877.

12. Hughes JE, Stewart J, Barclay GR, Govan JRW. Priming of neutrophil respiratory burst activity by lipopolysaccharide from Burkholderia cepacia. Infect Immun 1997; 65: 4281-4287.

13. Zughaier SM, Ryley HC, Jackson SK. Lipopolysaccharide (LPS) from Burkholderia cepacia is more active than LPS from Pseudomonas aeruginosa and Stenotrophomonas maltophilia in stimulating tumor necrosis factor alpha from human monocytes. Infect Immun 1999; 67: 1505-1507.

14. McKevitt AI, Bajaksouzian S, Klinger JD, Woods DE. Purification and characterization of an extracellular protease from Pseudomonas cepacia. Infect Immun 1989; 57: 771-778.

15. Lonon MK, Woods DE, Straus DC. Production of lipase by clinical isolates of Pseudomonas cepacia. J Clin Microbiol 1988; 26: 979-984

16. Zughaier SM, Ryley HC, Jackson SK. A melanin pigment purified from an epidemic strain of Burkholderia cepacia attenuates monocyte respiratory burst activity by scavenging superoxide anion. Infect Immun 1999; 67: 908-913.

17. Sajjan US, Forstner JF. Identification of the mucin-binding adhesin of isolated Pseudomonas cepacia from patients with cystic fibrosis. Infect Immun 1992; 60: 1434-1440.

18. Sajjan US, Forstner JF. Role of a 22-kilodalton pilin protein in binding of Pseudomonas cepacia to buccal epithelial cells Infect Immun 1993; 61: 3157-3163.

19. Sajjan US, Sylvester FA, Forstner JF. Cable-piliated Burkholderia cepacia binds to cytokeratin 13 of epithelial cells. Infect Immun 2000; 68: 1787-1795.
20. Sajjan US, Wu Y, Kent G, Forstner J. Preferential adherence of cable-piliated Burkholderia cepacia to respiratory epithelia of $\mathrm{CF}$ knockout mice and human cystic fibrosis lung explants. $J$ Med Microbiol 2000; 49: 875-885.

21. Saini LS, Galsworthy SB, John MA, Valvano MA. Intracellular survival of Burkholderia cepacia complex isolates in the presence of macrophage cell activation. Microbiology 1999; 145: $3465-3475$.

22. Martin DW, Mohr CD. Invasion and intracellular survival of Burkholderia cepacia. Infect Immun 2000; 68: 24-29.

23. Burns JL, Jonas M, Chi EY, Clark DK, Berger A, Griffith A. Invasion of respiratory epithelial cells by Burkholderia (Pseudomonas) cepacia. Infect Immun 1996; 64: 4054-4059.

24. Baird RM, Brown H, Smith AW, Watson ML. Burkholderia cepacia is resistant to the antimicrobial activity of airway epithelial cells. Immunopharmacology 1999; 44: 267-272.

25. Sylvester FA, Sajjan US, Forstner JF. Burkholderia (basonym Pseudomonas) cepacia binding to lipid receptors. Infect Immun 1996; 64: 1420-1425.

26. Shi S-R, Key ME, Kalra KL. Antigen retrieval in formalinfixed, paraffin-embedded tissues: an enhancement method for immunohistochemical staining based on microwave oven heating of tissue sections. J Histochem Cytochem 1991; 39: $741-748$.

27. Tomashefski JF, Thomassen M-J, Bruce MC, Goldberg HI, Konstan MW, Stern RC. Pseudomonas cepacia-associated pneumonia in cystic fibrosis. Arch Pathol Lab Med 1988; 112: $166-172$

28. Johansen HK, Kovesi TA, Koch C, Corey M, Hoiby N, Levison H. Pseudomonas aeruginosa and Burkholderia cepacia infection in cystic fibrosis patients treated in Toronto and Copenhagen. Pediatr Pulmonol 1998; 26: 89-96.

29. Isles A, Maclusky I, Corey M et al. Pseudomonas cepacia infection in cystic fibrosis: an emerging problem. $J$ Pediatr 1984; 104: 206-210.

30. Frangolias DD, Mahenthiralingam E, Rae S et al. Burkholderia cepacia in cystic fibrosis: variable disease course. Am J Respir Crit Care Med 1999; 160: 1572-1577.

31. Tablan OC, Chorba TL, Schidlow DV et al. Pseudomonas cepacia colonization in patients with cystic fibrosis: risk factors and clinical outcome. J Pediatr 1985; 107: 382-387.

32. Chaparro C, Gutierrez CA, Chan C, Tullis E, Hutcheon M, Keshavjee S. Lung transplantation for cystic fibrosis: the Toronto experience. In: Thirteenth Annual North American Cystic Fibrosis Conference. Seattle, Washington. Pediatr Pulmonol 1999; Suppl 19: Abstract 450.

33. Snell GI, de Hoyos A, Krajden M, Winton T, Mauere JR. Pseudomonas cepacia in lung transplant recipients with cystic fibrosis. Chest 1993; 103: 466-471.

34. Ledson MJ, Gallagher MJ, Corkill JE, Hart CA, Walshaw MJ. Cross infection between cystic fibrosis patients colonised with Burkholderia cepacia. Thorax 1998; 53: 432-436.

35. Kaplan SL, Mason EO, Wiedermann BL. Role of adherence in the pathogenesis of Haemophilus influenzae type $\mathrm{b}$ infection in infant rats. Infect Immun 1983; 42: 612-617.

36. Alkan ML, Wong L, Silverblatt FJ. Change in degree of type piliation of Escherichia coli during experimental peritonitis in the mouse. Infect Immun 1986; 54: 549-554. 